\section{Response to Plon et al.-Genetic testing and cancer risk management recommendations by physicians for at-risk relatives}

\section{To the Editor:}

This study ${ }^{1}$ represents a valuable effort to provide insight into the ability of physicians in specialties other than genetics to appropriately use genetic testing in their practices. Two significant sources of concern were identified, based on the responses of participating physicians to case-based scenarios describing the outcome of testing for mutations in BRCA1 and BRCA2. First, a small minority of responding physicians seem to have interpreted a variant of uncertain significance (VUS) as equivalent to a deleterious mutation. Second, some of the responding physicians indicated that they would order unnecessary Comprehensive full sequencing for an unaffected relative of a patient in whom a VUS or deleterious mutation was detected.

An important consideration relevant to the findings in this study is the potential positive impact of laboratory protocols that proactively address the areas of concern studied in this article. As a representative of Myriad Genetic Laboratories, Inc., the clinical laboratory that performs almost all of the BRCA1/2 testing in the United States, I would like to describe existing practices at Myriad relevant to the finding of this study and suggest that without taking this into account, the survey methodology may have led to an overstatement of the extent to which there is an adverse impact on clinical outcomes in practice.

\section{Management of patients with VUS}

The current BRCA1/2 VUS rate for testing performed by Myriad in the United States is $5 \%$, so it is likely than an individual provider will order a great many tests before receiving their first VUS. This study did not examine the differences in response based upon whether a physician specifically had past experience with a VUS result. This is important because, in the case of BRCA1/2 testing at Myriad, Medical Affairs staff reviews all VUS reports to determine if this is the first such result being sent to a provider. If so, the provider is contacted by either a Salt Lake City based or local genetic counselor or oncology nurse medical specialist. The primary goal of these contacts is to ensure a correct interpretation of the results, bearing in mind that there are cases where high-risk medical management is appropriate depending on the patient's personal and family history. These contacts also provide an opportunity to explain the options available for participation in Myriad's program to gather additional data for variants through family testing and to emphasize that it is not appropriate to test for a VUS in unaffected relatives for clinical purposes.

\section{Ordering comprehensive testing}

Myriad has protocols in place to determine whether there is any indication whatsoever that a mutation has been previously detected in a relative of the patient. This could be a notation on a requisition form, mention in a letter of medical necessity, a copy of a relative's lab report, or a verbal communication with an office or patient during the insurance preauthorization process. If a case is flagged for this reason, the provider is contacted by a Myriad genetic counselor for clarification before the Comprehensive testing is started. All cases are reviewed a second time by Medical Affairs staff before results are reported, to further identify and follow-up on cases for which single-site testing would have been more appropriate. Myriad staff currently contacts providers on approximately $2 \%$ of all Comprehensive $B R C A 1 / 2$ test orders to resolve this issue, which we estimate results in a change to the test request a third to one half of the time. With this in mind, it seems likely that the number of survey respondents who stated that they would order inappropriate Comprehensive testing is not an accurate reflection of the number of times that such testing is actually run.

As the use of personalized medicine expands, the Secretary's Advisory Committee on Genetics, Health and Society has recognized that "the ability of the genetics-specific health care workforce is not sufficient to meet the need ... and the large and diverse group of health professionals providing services at the point of care must be enlisted to provide appropriate genetic services and information." Issues raised in this article should continue to be a focus of educational programs, in conjunction with proactive laboratory practices such as those used at Myriad.

Eric Rosenthal, PhD, ScM Myriad Genetic Laboratories, Inc. Salt Lake City, Utah

Disclosure: The author is an employee and stockholder in Myriad Genetics.

\section{REFERENCE}

1. Plon SE, Cooper HP, Parks B, et al. Genetic testing and cancer risk management recommendations by physicians for at-risk relatives. Genet Med 2011; $13: 148-154$. 